\title{
A bifunctional O-GlcNAc transferase governs flagellar motility through anti-repression
}

\author{
Aimee Shen, ${ }^{1}$ Heather D. Kamp, ${ }^{1}$ Angelika Gründling, ${ }^{2}$ and Darren E. Higgins ${ }^{3}$ \\ Department of Microbiology and Molecular Genetics, Harvard Medical School, Boston, Massachusetts 02115, USA
}

Flagellar motility is an essential mechanism by which bacteria adapt to and survive in diverse environments. Although flagella confer an advantage to many bacterial pathogens for colonization during infection, bacterial flagellins also stimulate host innate immune responses. Consequently, many bacterial pathogens down-regulate flagella production following initial infection. Listeria monocytogenes is a facultative intracellular pathogen that represses transcription of flagellar motility genes at physiological temperatures $\left(37^{\circ} \mathrm{C}\right.$ and above). Temperature-dependent expression of flagellar motility genes is mediated by the opposing activities of MogR, a DNA-binding transcriptional repressor, and DegU, a response regulator that functions as an indirect antagonist of MogR. In this study, we identify an additional component of the molecular circuitry governing temperature-dependent flagellar gene expression. At low temperatures $\left(30^{\circ} \mathrm{C}\right.$ and below), MogR repression activity is specifically inhibited by an anti-repressor, GmaR. We demonstrate that GmaR forms a stable complex with MogR, preventing MogR from binding its DNA target sites. GmaR anti-repression activity is temperature dependent due to DegU-dependent transcriptional activation of gmaR at low temperatures. Thus, GmaR production represents the first committed step for flagella production in L. monocytogenes. Interestingly, GmaR also functions as a glycosyltransferase exhibiting O-linked $\mathrm{N}$-acetylglucosamine transferase (OGT) activity for flagellin (FlaA). GmaR is the first OGT to be identified and characterized in prokaryotes that specifically $\beta-O-G l c N A c y l a t e s$ a prokaryotic protein. Unlike the well-characterized, highly conserved OGT regulatory protein in eukaryotes, the catalytic activity of GmaR is functionally separable from its anti-repression function. These results establish GmaR as the first known example of a bifunctional protein that transcriptionally regulates expression of its enzymatic substrate.

[Keywords: Flagella; Listeria monocytogenes; MogR; DegU; GmaR; temperature-dependent regulation; O-linked GlcNAc transferase]

Supplemental material is available at http://www.genesdev.org.

Received September 12, 2006; revised version accepted October 23, 2006.

Flagellar motility is a fundamental mechanism by which bacteria acquire nutrients, colonize surfaces, and establish infections. Although flagellar motility confers a growth advantage in many environments, production of flagella is a complex, energy-demanding developmental process and thus is exquisitely regulated in response to many environmental cues (Aldridge and Hughes 2002; Macnab 2003). For example, flagella can enhance adherence and invasion in the early stages of host infection, yet continuous production of flagella during infection can stimulate innate immune responses (Hayashi et al. 2001; Molofsky et al. 2006; Ren et al. 2006) or impede subsequent colonization events (for review, see Ramos et

\footnotetext{
${ }^{1}$ These authors contributed equally to this work.

${ }^{2}$ Present address: Department of Microbiology, University of Chicago, Chicago, IL 60637, USA.

${ }^{3}$ Corresponding author.

E-MAIL dhiggins@hms.harvard.edu; FAX (617) 738-7664.

Article is online at http://www.genesdev.org/cgi/doi/10.1101/gad.1492606.
}

al. 2004). Thus, many facultative bacterial pathogens down-regulate production of flagella shortly after infection (Akerley et al. 1995; Hughes and Galan 2002). A primary environmental cue that initiates repression of flagellar gene transcription during infection is physiological temperature $\left(37^{\circ} \mathrm{C}\right)$ (Ott et al. 1991; Akerley and Miller 1993; Kapatral et al. 1996).

Listeria monocytogenes is a food-borne facultative intracellular pathogen that down-regulates flagellar gene expression upon encountering physiological temperatures $\left(37^{\circ} \mathrm{C}\right.$ and above) (Peel et al. 1988). While flagellar motility is essential for L. monocytogenes biofilm formation and persistence in specific environments, such as food processing plants (Vatanyoopaisarn et al. 2000), constitutive expression of flagellar genes during infection attenuates the virulence of L. monocytogenes (Gründling et al. 2004). Recent studies have partially characterized the molecular circuitry governing temperature-dependent flagellar gene expression in L. monocytogenes. At 
physiological temperatures, MogR, a transcriptional repressor, inhibits flagellar gene transcription by directly binding to flagellar gene promoters (Gründling et al. 2004; Shen and Higgins 2006). Interestingly, MogR protein levels are temperature independent (Shen and Higgins 2006), suggesting that post-translational regulation of MogR function permits flagellar gene expression at low temperatures $\left(30^{\circ} \mathrm{C}\right.$ and below $)$. Modulation of MogR repression activity at low temperatures is mediated by a response regulator, DegU (Shen and Higgins 2006). Although expression of DegU is required for flagellar gene transcription at low temperatures (Knudsen et al. 2004; Williams et al. 2005), DegU is largely dispensable for flagellar gene transcription in the absence of MogR (Shen and Higgins 2006). Thus, DegU, or a DegUregulated factor, antagonizes $\mathrm{Mog}$ repression activity at low temperatures.

Expression of the flagellin subunit (FlaA) is also regulated at the post-transcriptional and post-translational level in L. monocytogenes. Maximal FlaA protein production requires DegU, since MogR-, DegU-negative bacteria overexpress flaA transcripts but fail to produce wild-type levels of FlaA protein (Shen and Higgins 2006). Thus, DegU regulates FlaA expression at both the transcriptional and post-transcriptional level. FlaA also undergoes a post-translational modification. FlaA subunits are covalently modified by monomeric $\beta$-O-linked $\mathrm{N}$ acetylglucosamine (GlcNAc) residues at three to six sites per subunit (Schirm et al. 2004). The functional consequence of flagellin glycosylation in L. monocytogenes, or in any Gram-positive bacterium, remains unknown. In many Gram-negative bacteria, flagellin glycosylation regulates its secretion and modulates host immune responses against flagellin (Logan et al. 2002; Takeuchi et al. 2003; Verma et al. 2005; Logan 2006). Nevertheless, the observation that FlaA is modified by $\beta$-O-linked GlcNAc residues indicates that $L$. monocytogenes encodes an enzyme with $\beta$-O-linked GlcNAc transferase (OGT) activity for FlaA. Whereas no bacterial enzyme with OGT activity for a prokaryotic protein has been characterized to date (Schirm et al. 2004), the highly conserved OGT enzyme in eukaryotes covalently modifies numerous nuclear and cytoplasmic proteins to regulate processes ranging from apoptosis to insulin metabolism (for review, see Love and Hanover 2005).

In this report, we identify the first bacterial OGT specific for a prokaryotic protein as GmaR, a DegU-regulated flagellin glycosyltransferase that mediates $\beta$-O-linked GlcNAc modification of FlaA in L. monocytogenes. GmaR is required for flagellar motility, as GmaR-negative bacteria are nonmotile. This motility defect, however, is not due to a failure to secrete FlaA, as observed in glycosyltransferase mutants of other organisms, but rather due to a defect in flagellar gene transcription. Our studies indicate that GmaR permits flagellar gene expression at low temperatures by binding directly to MogR and inhibiting its ability to bind target sequences in flagellar gene promoters. Thus, GmaR also functions as an anti-repressor for MogR. We further demonstrate that DegU-dependent, temperature-regulated production of GmaR is the first committed step for flagellar elaboration in L. monocytogenes. Unlike OGT-mediated transcriptional regulation in eukaryotes, the OGT activity of GmaR is dispensable for its anti-repressor function. Thus, our findings reveal GmaR as the first example of a bifunctional glycosyltransferase that transcriptionally regulates the expression of its enzymatic substrate.

\section{Results}

\section{DegU regulates glycosylation of FlaA}

We previously demonstrated that deletion of $\operatorname{deg} U$ in MogR-negative bacteria $(\Delta \operatorname{mog} R \Delta \operatorname{deg} U)$ reduces FlaA levels without affecting flaA transcription (Shen and Higgins 2006). Post-transcriptional regulation of flagellin levels has previously been observed in other bacteria. In Helicobacter pylori, inactivation of flagellin glycosyltransferase genes severely decreases flagellin levels without altering transcription of the flagellin gene (Schirm et al. 2003). Glycosyltransferase mutants also exhibit diminished flagellin levels in Helicobacter felis, Caulobacter crescentus, Campylobacter sp., and Aeromonas sp. (for review, see Logan 2006), underscoring the role that glycosylation plays in regulating flagellin levels. Since L. monocytogenes FlaA is heavily glycosylated, and glycosyltransferase mutants in other bacteria are defective in flagellin production, we reasoned that glycosylation of FlaA in L. monocytogenes might similarly be required for maximal FlaA production. Specifically, we hypothesized that the reduced levels of FlaA observed in $\Delta \operatorname{mog} R \Delta \operatorname{deg} U$ might be attributable to a defect in FlaA glycosylation. To examine this possibility, $\beta$-O-linked GlcNAc modification of FlaA in $\Delta \operatorname{mog} R \quad \Delta \operatorname{deg} U$ and $\Delta m o g R$ was compared by Western blot analysis using an antibody specific for $\beta$-O-linked GlcNAc (Comer et al. 2001). If DegU regulates FlaA glycosylation, the proportion of $\beta$-O-linked GlcNAc modification of FlaA should be lower in $\Delta \operatorname{mog} R \Delta \operatorname{deg} U$ relative to $\Delta \operatorname{mog} R$, in addition to $\Delta \operatorname{mog} R \Delta \operatorname{deg} U$ producing less FlaA than $\Delta \operatorname{mog} R$ (Supplementary Fig. S1, left panel; Shen and Higgins 2006). Comparison of total cell-wall-associated FlaA to $\beta$-O-linked GlcNAc-modified FlaA revealed that the proportion of modified FlaA in $\Delta \operatorname{mog} R \Delta \operatorname{deg} U$ was lower than that of $\Delta \operatorname{mog} R$ (Supplementary Figure S1, cf. left and right panels), suggesting that the absence of DegU expression impairs FlaA glycosylation.

\section{Lmo0688 is required for flaA transcription and FlaA glycosylation}

To further explore the relationship between FlaA glycosylation and FlaA production in $\Delta \operatorname{mog} R \Delta \operatorname{deg} U$, we attempted to identify the putative DegU-regulated glycosyltransferase responsible for modifying FlaA. Since all characterized flagellin glycosyltransferase genes are located within close proximity to the gene encoding flagellin (Logan 2006), we searched the flagellar motility locus of $L$. monocytogenes for genes encoding glycosyltrans- 
ferase domains. 1 mo0688 was the only gene in this locus predicted to encode a glycosyltransferase. Significantly, Imo0688 is DegU-regulated (Williams et al. 2005) and was identified by our group as being required for motility by transposon mutagenesis (data not shown). Since Lmo0688 appeared to be a DegU-regulated glycosyltransferase, we examined the effect of deleting Imo0688 on FlaA glycosylation and FlaA protein levels.

Consistent with the results of transposon mutagenesis, an in-frame deletion mutant of 1 mo0688 ( $\Delta 688)$ resulted in nonmotile bacteria (Fig. 1A). Given that flagellar glycosyltransferase mutants of Helicobacter sp. and Campylobacter sp. are nonmotile due to a failure to secrete unglycosylated FlaA (Josenhans et al. 2002; Goon et al. 2003), we examined FlaA levels in cellular fractions of $\Delta 688$ by Western blot analysis. FlaA was undetectable in either whole-cell lysates (Fig. 1B) or cytoplasmic, cellwall-associated, or supernatant fractions of $\Delta 688$ (data not shown). The defect in motility and FlaA production of $\Delta 688$ was solely attributable to the absence of Lmo0688, since heterologous expression of 1 mo0688 from an ectopic locus in $\Delta 688$ bacteria $(\Delta 688 / \mathrm{c} 688)$ restored motility and FlaA production (Fig. 1A,B). To determine if a transcriptional or post-transcriptional mechanism was responsible for the complete absence of detectable FlaA, we examined flaA transcription in $\Delta 688$. Surprisingly, flaA transcripts were undetectable in $\Delta 688$ by Northern blot analysis (Fig. 1C) and reporter assays using fusions of the flaA promoter to lacZ (data not shown). Thus, our results indicate that a putative FlaA glycosyltransferase, Lmo0688, plays an unexpected role in regulating transcription of the gene encoding its substrate, flaA.

The finding that flaA transcription requires Lmo0688 expression raised the possibility that Lmo0688 might regulate transcription of other flagellar genes. Transcriptional profiling of $\Delta 688$ revealed that transcription of all known flagellar motility genes was repressed in $\Delta 688$ relative to wild type during growth at room temperature in BHI broth (Supplementary Table S1). Since MogR is known to directly repress transcription of all flagellar motility genes at $37^{\circ} \mathrm{C}$ (Shen and Higgins 2006), we hypothesized that Lmo0688 might permit flagellar gene transcription at low temperatures by antagonizing MogR repression activity. Therefore, Lmo0688 would be dispensable for flagellar gene expression in the absence of MogR. Indeed, deletion of $\operatorname{mog} R$ in $\Delta 688(\Delta \operatorname{mog} R \Delta 688)$ restored transcription of flaA (Fig. 1C) and other motility genes (data not shown), FlaA production (Fig. 1B), and motility (Fig. 1A), indicating that Lmo0688 antagonizes MogR-mediated repression of flagellar gene transcription. However, Lmo0688 was still required for glycosylation of FlaA, as FlaA produced by $\Delta \operatorname{mog} R \Delta 688$ migrated at a lower apparent molecular weight than $\Delta \operatorname{mog} R$ by SDS-PAGE and was not $\beta$-O-GlcNAcylated (Fig. 1D). Taken together, these results indicate that Lmo0688 functions dually to antagonize MogR-mediated repression of flagellar motility gene transcription and to glycosylate FlaA.

\section{Lmo0688 is the DegU-regulated factor that antagonizes $\operatorname{Mog} R$ repression activity}

Since deletion of $\operatorname{mog} R$ in either $\Delta 688$ or $\Delta \operatorname{deg} U$ bacteria restores FlaA expression (Fig. 1; Supplementary Fig. S1), we hypothesized that DegU and Lmo0688 are similarly required to alleviate MogR repression of flagellar motility gene transcription. To explore this possibility, we compared the transcriptional profiles of $\Delta 688, \Delta \operatorname{deg} U$, and $\Delta \operatorname{mog} R$ relative to wild type using hierarchal cluster analysis, which groups together coordinately regulated genes in an unsupervised manner (Eisen et al. 1998). Flagellar motility genes grouped together naturally by cluster analysis and were reciprocally regulated in a temperature-dependent manner by MogR (repressed at $37^{\circ} \mathrm{C}$ ) and DegU/Lmo0688 (activated at room temperature) (Fig. 2).
A

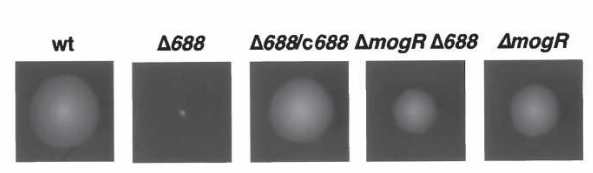

C

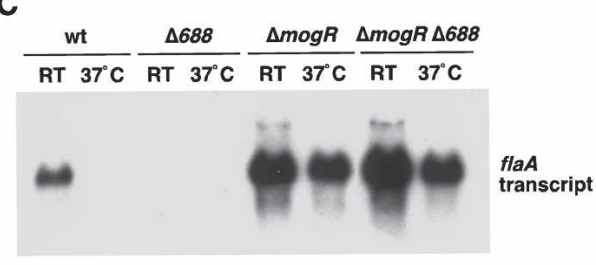

B

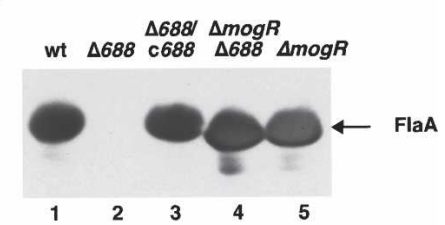

D

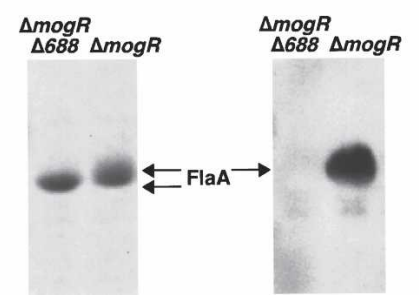

Figure 1. Lmo0688 is required to alleviate $\mathrm{Mog}$ R repression of flaA transcription at low temperatures and to glycosylate FlaA. (A) Motility analysis of $L$. monocytogenes strains. A single colony of wild type (wt), $\Delta 688, \Delta 688$ / c688, $\Delta \operatorname{mog} R \Delta 688$, and $\Delta \operatorname{mog} R$ was inoculated in low-agar $(0.375 \%) \mathrm{BHI}$ plates and incubated for $48 \mathrm{~h}$ at room temperature. Strain $\Delta 688 / \mathrm{c} 688$ contains a complementing 1 mo0688 expressed from a heterologous promoter in an ectopic chromosomal locus. (B) Analysis of FlaA protein in whole-cell lysates of strains used in $A$. L. monocytogenes cultures were grown in $\mathrm{BHI}$ broth for $20 \mathrm{~h}$ at room temperature. Lysates were separated by SDS-PAGE and analyzed by Western blot using a FlaA-specific antibody. (C) Northern blot analysis of flaA transcripts in selected strains used in A. L. monocytogenes cultures were grown in $\mathrm{BHI}$ broth at room temperature or $37^{\circ} \mathrm{C}$, and RNA was harvested after $20 \mathrm{~h}$. (D) Analysis of $\beta$-O-linked glycosylation in cell-wall-associated fractions of $\Delta \operatorname{mog} R \Delta 688$ and $\Delta \operatorname{mog} R$. L. monocytogenes cultures were grown in BHI broth for $20 \mathrm{~h}$ at room temperature. Cell-wall-associated FlaA was resolved by SDS-PAGE and detected by Coomassie stain (left panel) and Western blot using a $\beta$-O-linked GlcNAc-specific antibody (right panel). Differential mobility of FlaA by SDS-PAGE is noted (arrows). 
Shen et al.

Figure 2. DegU and Lmo0688 have similar roles in antagonizing MogR repression of flagellar motility gene expression. Cluster display of microarray analyses of $\Delta 688$ (Lmo0688 regulon), $\Delta \operatorname{deg} U$ (DegU regulon), and $\Delta \operatorname{mog} R$ (MogR regulon) compared with wild type grown in $\mathrm{BHI}$ broth at room temperature or $37^{\circ} \mathrm{C}$. (Far left) Relationships among genes are represented by a dendrogram, where the branch lengths reflect the degree of similarity between gene expression patterns. The color scale ranges from saturated green for fold ratios 5.0 and below to saturated red for fold ratios 5.0 and above. Green represents genes that are activated in wild type relative to the mutant, red represents genes that are repressed in wild type relative to the mutant, and black represents genes for which no difference in expression was observed between the mutant and wild type. Gray indicates genes for which the hybridization data was too poor to be included in the analysis.

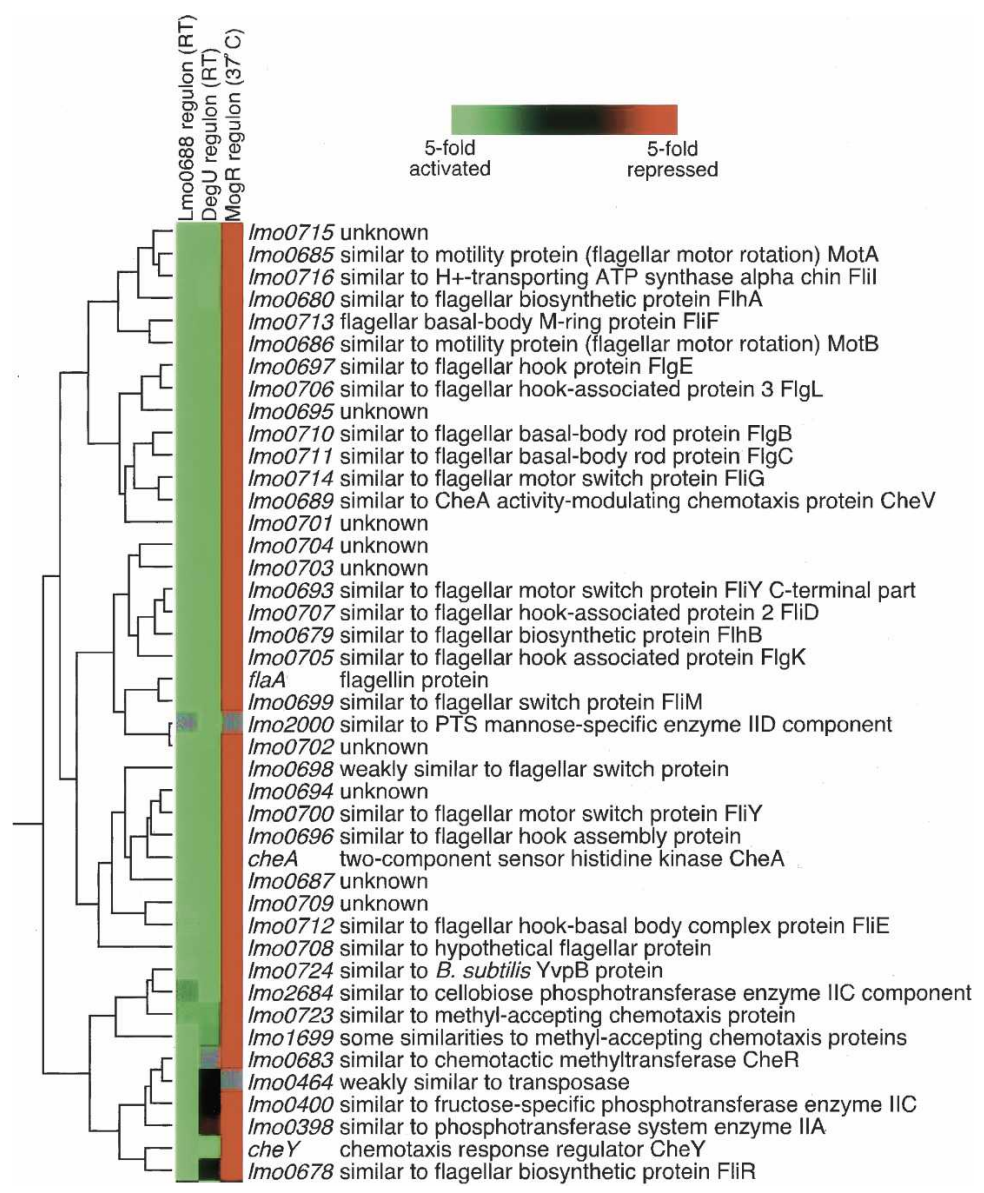

observed in $\Delta$ degU/c688, FlaA protein levels were significantly reduced compared with wild type, and $\Delta d e g U /$ c688 bacteria were nonmotile (Fig. 3B,C). This result is consistent with our previous observation that DegU is required for maximizing FlaA production at both a transcriptional and post-transcriptional level (Shen and Higgins 2006).

\section{The glycosyltransferase activity of Lmo0688} is dispensable for its anti-repressor function

Although our data indicated that Lmo0688 is sufficient to antagonize $\operatorname{Mog} \mathrm{R}$ repression activity, the mechanism by which Lmo0688 functions as an anti-repressor remained unclear. Bioinformatic analyses of Lmo0688 predicted the presence of a Family 2 glycosyltransferase domain and three tetratricopeptide repeat (TPR) domains (Fig. 4A). TPR domains have been shown to mediate protein-protein interactions and modulate the substrate specificity of glycosyltransferase domains (Blatch and Lassle 1999; Lubas and Hanover 2000; Iyer and Hart 2003). Intriguingly, a link between glycosyltransferase activity and transcriptional regulation has long been recognized in eukaryotes (for review, see Love and Hanover 2005). OGT-mediated dynamic $\beta$-O-linked GlcNAc glycosylation of transcription factors, RNA polymerase II, and even the proteasome regulates both transcriptional 
A

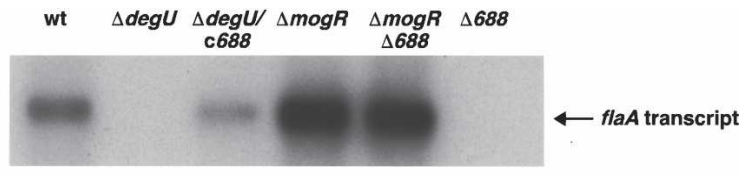

B

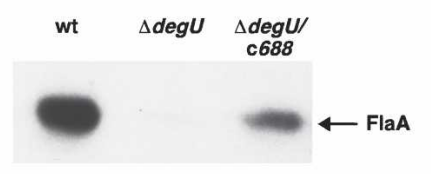

C

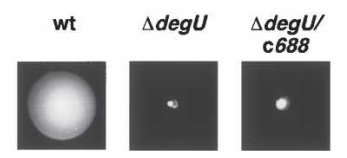

Figure 3. Lmo0688 is the DegU-regulated factor that antagonizes MogR repression activity. (A) Northern blot analysis of flaA transcript levels. RNA was harvested from $L$. monocytogenes strains wild type (wt), $\Delta \operatorname{deg} U, \Delta \operatorname{deg} U / \mathrm{c} 688, \Delta \operatorname{mog} R$, $\Delta \operatorname{mog} R \Delta 688$, and $\Delta 688$ following growth for $20 \mathrm{~h}$ in BHI broth at room temperature. $(B)$ Western blot analysis of FlaA protein levels. Cultures were grown $6 \mathrm{~h}$ in BHI broth at room temperature. Whole-cell lysates were analyzed using a FlaA-specific antibody. (C) Motility analysis of strains used in $B$. A single colony was inoculated in $0.375 \%$ BHI agar and incubated for $48 \mathrm{~h}$ at room temperature.

repression and activation (Jackson and Tjian 1988; Yang et al. 2002; Zhang et al. 2003). Since Lmo0688 and OGT share identical enzymatic functions, we examined the possibility that the putative glycosyltransferase activity of Lmo0688 is required for its anti-repression function.

To first assess glycosyltransferase activity of Lmo0688, we determined whether purified $\mathrm{His}_{6}$-tagged Lmo0688 could O-GlcNAcylate its predicted substrate, FlaA. When purified Lmo0688 was incubated with whole-cell lysates of $\Delta \operatorname{mog} R \Delta 688$ bacteria, which contained unglycosylated FlaA, Lmo0688 mediated transfer of $\left[{ }^{14} \mathrm{C}\right]$ UDP-GlcNAc to an $\sim 30-k D a$ protein (Fig. 4C, lanes 5-8). In contrast, glycosylation of this $\sim 30-\mathrm{kDa}$ protein was not observed upon incubation of purified Lmo0688 with whole-cell extracts of $\Delta \operatorname{mog} R \Delta 688 \Delta f l a A$ bacteria, implying that the $\sim 30-\mathrm{kDa}$ modified protein is FlaA and that FlaA is the primary substrate of Lmo0688 (Fig. 4C, lanes 1-4). $\beta$-O-linked GlcNAc modification of FlaA could also be detected upon incubation of purified Lmo0688 with extracts prepared from Escherichia coli heterologously expressing $L$. monocytogenes FlaA (data not shown). Since $E$. coli lacks endogenous OGT activity (Lubas and Hanover 2000), this latter result strongly suggests that Lmo0688 directly mediates O-GlcNAcylation of FlaA. In contrast, O-linked modification of MogR could not be detected by immunoprecipitation and Western blot analyses or with the in vitro glycosylation assay (data not shown)

Given that Lmo0688 has glycosyltransferase activity, we examined whether this activity was required for Lmo0688 to function as an anti-repressor. To this end, we inactivated the catalytic function of Lmo0688 and examined the effect of this mutation on FlaA expression.
Since Family 2 glycosyltransferases are defined by an invariant $\mathrm{DxD}$ motif that constitutes their active site (Campbell et al. 1997; Unligil et al. 2000), we aligned Lmo0688 with 12 bacterial Family 2 glycosyltransferases to identify the DxD motif of Lmo0688 (amino acids 8385) (Fig. 4B). Incubation of purified $\mathrm{His}_{6}$-tagged Lmo0688 carrying active site mutations (D83N D85N) with cell extracts prepared from $\Delta \operatorname{mog} R \Delta 688$ bacteria confirmed that the DxD motif is essential for catalytic function (Fig. 4C, lanes 9-12). When these active site mutations were introduced into $1 \mathrm{mo} 0688$ in its native locus within L. monocytogenes $\left(688^{\star}\right)$, flagellar motility remained intact (Fig. 4D), although FlaA glycosylation was ablated (Fig. 4E). Thus, the glycosyltransferase activity of Lmo0688 is not required to antagonize MogR repression function, indicating that the catalytic and regulatory activities of Lmo0688 are functionally distinct. In addition, the observation that $688^{*}$ was fully motile (Fig. 4D) demonstrates that flagellin glycosylation does not affect the production, assembly, or function of flagella in L. monocytogenes as previously suggested.

\section{Lmo0688 removes $\operatorname{Mog} R$ bound to flaA promoter region DNA by protein-protein interaction}

Since the glycosyltransferase activity of Lmo0688 is not required for its anti-repressor function, it is probable that regions outside of the glycosyltransferase domain function to antagonize MogR repression activity. Although Lmo0688 lacks a predicted DNA-binding motif, domain analysis revealed a TPR repeat region (Fig. 4A) that has been previously implicated in protein-protein interactions (Blatch and Lassle 1999; Iyer and Hart 2003). We showed recently that MogR represses transcription of all known flagellar motility genes by directly binding to TTTT- $\mathrm{N}_{5}$-AAAA sites in flagellar promoter region DNA (Shen and Higgins 2006). It is possible that Lmo0688 interferes with $\operatorname{Mog} \mathrm{R}$ repression activity by (1) binding DNA sequences that overlap MogR target sites and occluding MogR binding, (2) interacting directly with MogR and altering its ability to bind promoter region DNA, or (3) interacting with another factor that is not DegU regulated, but is present at low temperatures, to inhibit MogR binding to promoter region DNA.

To distinguish these possibilities, we examined whether Lmo0688 altered the ability of MogR to bind to its target sequences by gel mobility shift analysis. Incubation of radiolabeled flaA promoter region DNA with purified $\mathrm{His}_{6}$-tagged MogR resulted in the formation of shifted, supershifted and supersupershifted DNA complexes (Fig. 5A, lane 2; Shen and Higgins 2006). These shifted DNA complexes disappeared when increasing amounts of purified $\mathrm{His}_{6}$-tagged Lmo0688 were added to a constant amount of purified $\mathrm{His}_{6}$-tagged MogR (40 nM) previously bound to flaA promoter region DNA (Fig. 5A, lanes 3-6). Since Lmo0688 alone failed to bind and shift flaA promoter region DNA (Fig. 5A, lanes 8-11), these results suggest that Lmo0688 directly interacts with MogR and not flaA promoter region DNA. Importantly, $\mathrm{MogR}$ retained its ability to bind and shift flaA promoter 
Shen et al.

A

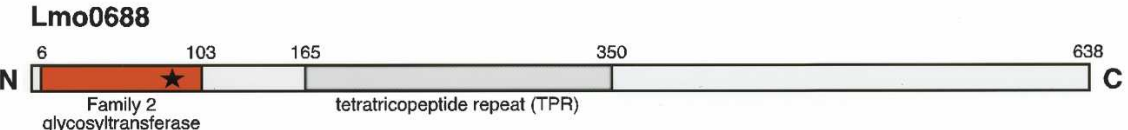

B

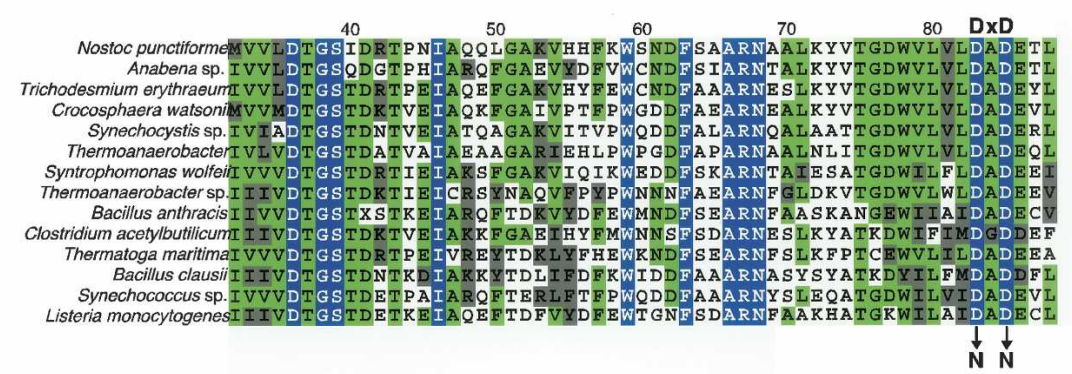

C
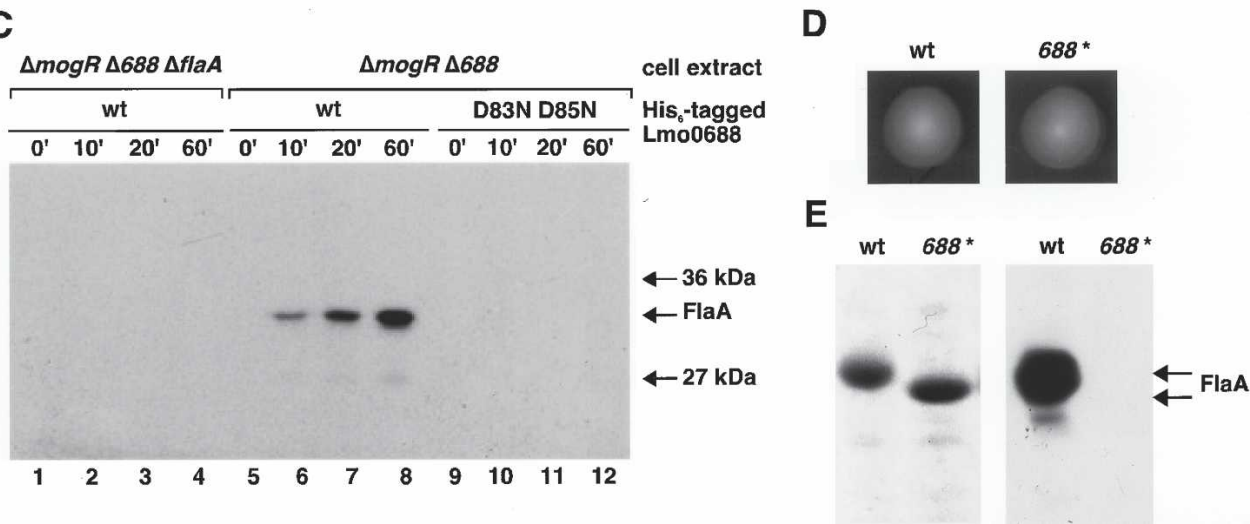

Figure 4. The glycosyltransferase activity of Lmo0688 is dispensable for FlaA production. (A) Schematic of Lmo0688 protein. The predicted glycosyltransferase domain (amino acids 6-103) is shown in red, and the DxD active site motif (amino acids 83-85) is represented as a black star. Three TPR domains (amino acids 165-350) are represented as a hatched box. The C-terminal domain lacks homology with known proteins. (B) Partial multiple sequence alignment of a subset of Lmo0688 homologs. Completely conserved identical residues are blocked in blue, conserved identical residues are blocked in green, and conserved similar residues are blocked in gray. The DxD glycosyltransferase motif is marked above the alignment, and the active site mutations in L. monocytogenes Lmo0688 (D83N D85N) are noted below the alignment. (C) In vitro glycosylation assay. L. monocytogenes strains $\Delta$ mogR $\Delta 688 \Delta f l a A$ and $\Delta \operatorname{mog} R \Delta 688$ were grown in BHI broth for $4 \mathrm{~h}$ at $28^{\circ} \mathrm{C}$ and mechanically lysed. Purified His ${ }_{6}$-tagged Lmo0688 (wild type or active site mutant, D83N D85N) was incubated with the indicated whole-cell lysates in the presence of $\left[{ }^{14} \mathrm{C}\right]-$ UDP-GlcNAc. O-GlcNAcylation was visualized by autoradiography. $(D)$ Motility analysis of wild type $(\mathrm{wt})$ and $688^{\star}$. A single colony was inoculated in $0.375 \%$ $\mathrm{BHI}$ agar and incubated for $48 \mathrm{~h}$ at room temperature. $688^{\star}$ carries the active site mutations (D83N D85N) that disrupt glycosyltransferase activity. (E) Analysis of FlaA glycosylation in strains used in D. L. monocytogenes strains were grown in BHI broth for $20 \mathrm{~h}$ at room temperature. FlaA protein levels in the cell-wall-associated fraction were resolved by SDS-PAGE and examined by Coomassie stain (left panel) and Western blot analysis using an $\beta$-O-linked GlcNAc-specific antibody (right panel).

region DNA in the presence of purified $\mathrm{His}_{6}$-tagged DegU (Fig. 5A, lane 7), indicating that Lmo0688 specifically mediates release of MogR from target DNA sequences. Furthermore, Lmo0688 was sufficient to inhibit MogR binding to cheY promoter region DNA, suggesting that Lmo0688 directly relieves MogR repression of all flagellar motility gene promoters (Supplementary Fig. S2A).

Since gel shift analysis suggested that Lmo0688 and MogR physically interact, we used affinity purification to determine if a direct and stable protein-protein interaction occurs between MogR and Lmo0688. Nickel affinity purification was performed with Ni-NTA agarose beads incubated with purified $\mathrm{His}_{6}$-tagged Lmo0688 and cell lysates prepared from L. monocytogenes strains.
Western blot analysis of affinity-purified binding reactions revealed that $\mathrm{His}_{6}$-tagged Lmo0688 associated with $\operatorname{Mog} R$ in lysates prepared from wild type and $\operatorname{imog} R$, a strain overexpressing $\operatorname{Mog} R$ under the control of an IPTG-inducible promoter (Fig. 5B, lanes 2,3). The interaction between Lmo0688 and MogR was specific, since MogR was not detected in the absence of $\mathrm{His}_{6}$-tagged Lmo0688 (Fig. 5B, lane 1). The reverse pull-down assay, using $\mathrm{His}_{6}$-tagged MogR and L. monocytogenes cell lysates expressing Lmo0688, specifically pulled down Lmo0688 (Supplementary Fig. S2B). Additional factors are not required for this interaction, since binding was observed between purified $\mathrm{His}_{6}$-tagged proteins using coimmunoprecipitation studies with a MogR-specific antibody, and analysis of pull-down reactions by Coomassie 


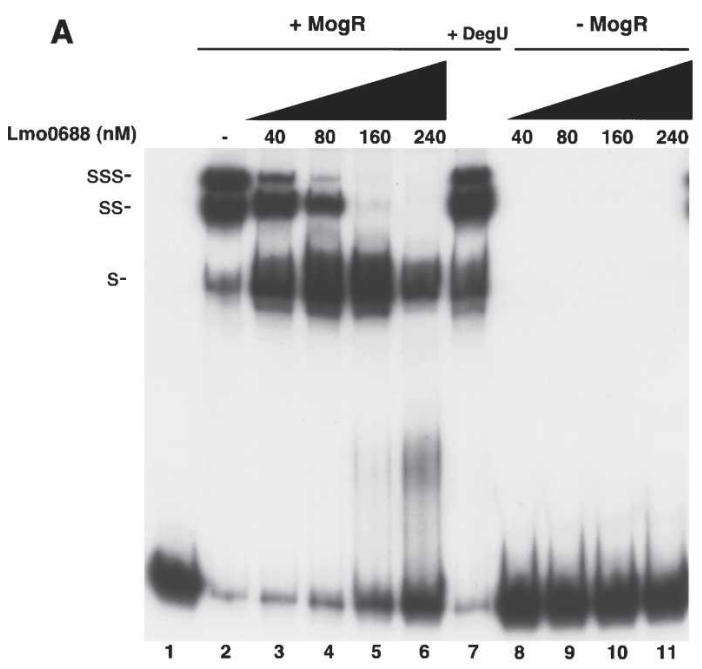

B

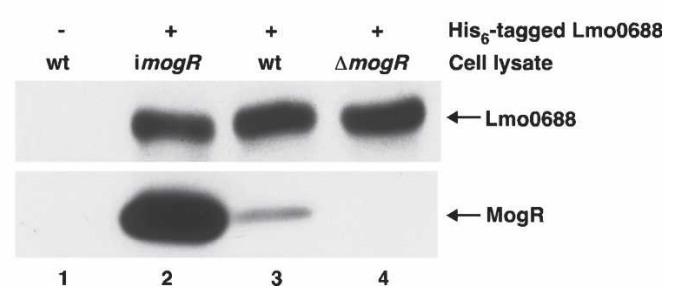

Figure 5. Lmo0688 removes $\mathrm{MogR}$ bound to flaA promoter region DNA by protein-protein interaction. $(A)$ Gel shift analysis of $\mathrm{MogR}$ and Lmo0688 binding to flaA promoter region DNA. Radiolabeled flaA promoter region DNA spanning -162 to +8 relative to the transcriptional start site was incubated with a constant amount (40 nM) of purified $\mathrm{His}_{6}$-tagged MogR to which increasing concentrations of His $_{6}$-tagged Lmo0688 (lanes 2-6) or $240 \mathrm{nM} \mathrm{His}{ }_{6}$-tagged DegU (lane 7) was added. (Lanes 8-11) Increasing concentrations of $\mathrm{His}_{6}{ }_{-}$-tagged Lmo0688 alone was incubated with radiolabeled fla $A$ promoter region DNA. The binding reactions were separated by nondenaturing PAGE and detected by autoradiography. Shifted (S), supershifted (SS), and supersupershifted (SSS) DNA complexes are indicated. (B) Pull-down assay of $\mathrm{MogR}$ by $\mathrm{Ni}^{2+}$ affinity purification of $\mathrm{His}_{6}$-tagged Lmo0688. Purified $\mathrm{His}_{6}$-tagged Lmo0688 was incubated with cell lysates prepared from $L$. monocytogenes strains wild type (wt), imogR, and $\Delta \operatorname{mog} R$. His ${ }_{6}$-tagged Lmo0688 and interacting proteins were isolated using Ni-NTA agarose beads. Proteins isolated in the pull-down assay were separated on a $10 \%$ SDS-PAGE gel and analyzed by Western blot using either a MogR- or Lmo0688-specific antibody.

stain failed to identify additional interacting proteins (data not shown). Taken together, our results indicate that a direct and stable protein-protein interaction occurs between Lmo0688 and MogR and that this specific interaction inhibits MogR repression of flagellar motility gene expression.

Temperature-dependent expression of Lmo0688 confers temperature specificity to flagellar motility gene transcription

We have previously shown that MogR continuously represses transcription of flagellar motility genes. How- ever, MogR repression is less stringent at low temperatures to permit flagella production and motility (Shen and Higgins 2006). Although our results indicate a mechanism by which Lmo0688 relieves MogR repression, the underlying mechanism governing temperature specificity for Lmo0688-mediated anti-repression remained unknown. To determine if the temperature regulation of $\operatorname{Mog} \mathrm{R}$ repression was due to changes in Lmo0688 expression, we examined 1 mo0688 transcript and Lmo0688 protein levels in L. monocytogenes cultures grown at either room temperature or $37^{\circ} \mathrm{C}$. Strikingly, Lmo0688 was detected only at room temperaure and not at $37^{\circ} \mathrm{C}$ in wild-type $L$. monocytogenes due to temperature-dependent transcription of 1 mo0688 (Fig. 6A,B). Expression of Lmo0688 appeared to be both DegUactivated and MogR-repressed (Fig. 6A), a result consistent with previous microarray studies (Williams et al. 2005; Shen and Higgins 2006). Lmo0688 was not detectable in $\Delta \operatorname{deg} U$ bacteria, while deletion of $\operatorname{mog} R$ in

\section{A}

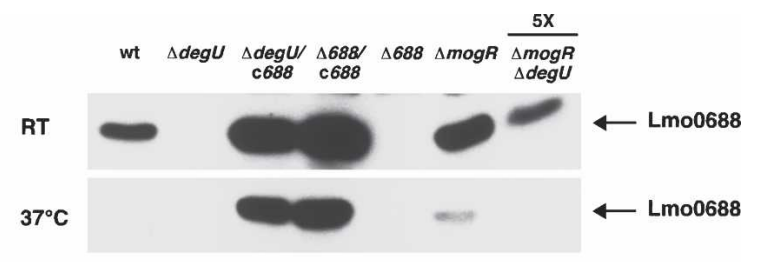

B

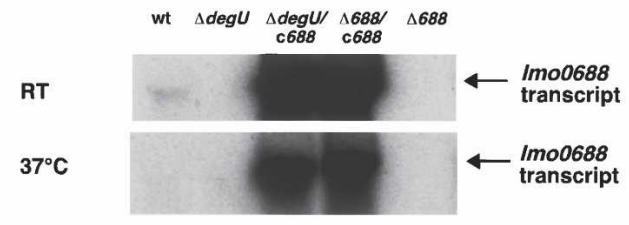

C

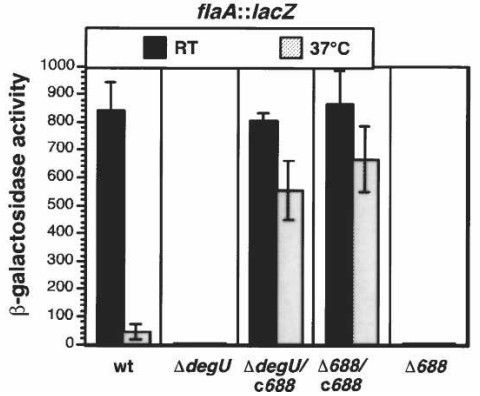

Figure 6. Temperature-dependent expression of Lmo0688 confers temperature specificity to flagellar motility gene transcription. (A) Western blot analysis of Lmo0688 in whole-cell lysates using an Lmo0688-specific antibody. L. monocytogenes strains wild type (wt), $\Delta \operatorname{deg} U, \Delta \operatorname{deg} U / \mathrm{c} 688, \Delta 688 / \mathrm{c} 688, \Delta 688, \Delta \operatorname{mog} R$, and $\Delta \operatorname{mog} R \Delta \operatorname{deg} U$ were grown for $6 \mathrm{~h}$ at room temperature or $37^{\circ} \mathrm{C}$ in BHI broth. Fivefold more sample was loaded for $\Delta \operatorname{mog} R \Delta \operatorname{deg} U$. (B) Northern blot analysis of 1 mo0688 transcript levels. RNA was harvested from strains grown in $A$. Blots were overexposed to detect the presence of 1 mo0688 transcript in the wild-type sample at room temperature. $(C)$ Analysis of flaA promoter activity determined by $\beta$-galactosidase assays. flaA ::Tn917 transposon insertion-derived strains were grown for $18-20 \mathrm{~h}$ at room temperature or $37^{\circ} \mathrm{C}$ in $\mathrm{BHI}$ broth. $\beta$-Galactosidase activities represent the means and standard deviations of three independent experiments. 
$\Delta$ deg $U$ bacteria $(\Delta \operatorname{mog} R \Delta \operatorname{deg} U)$ only partially restored Lmo0688 protein levels (Fig. 6A). Taken together, our results suggest that DegU-mediated activation of Imo0688 transcription at low temperatures allows for Lmo0688 production to relieve MogR-mediated repression of $1 \mathrm{mo0} 688$ and other flagellar motility genes.

If temperature regulation of MogR repression is simply due to the differential expression of Lmo0688, constitutive expression of Lmo0688 at a nonpermissive temperature $\left(37^{\circ} \mathrm{C}\right)$ should result in transcription of flagellar motility genes. Although $\mathrm{Mog}$ R stringently represses transcription of flaA at $37^{\circ} \mathrm{C}$ in wild-type bacteria, ectopic expression of $1 \mathrm{mo} 0688$ in $\Delta \mathrm{deg} U / \mathrm{c} 688$ and $\Delta 688 / \mathrm{c} 688$ resulted in robust transcription of $f l a A$ at $37^{\circ} \mathrm{C}$ as determined by $\beta$-galactosidase reporter assays (Fig. 6C). These results indicate that Lmo0688 can be biologically active as an anti-repressor at elevated temperatures. Nonetheless, despite elevated levels of flaA transcription at $37^{\circ} \mathrm{C}$, a defect in FlaA production and motility was still observed (data not shown). This result is consistent with prior studies indicating that, despite overexpressing fla $\mathrm{A}$ transcripts at $37^{\circ} \mathrm{C}, \Delta \operatorname{mog} R$ bacteria are nonmotile and produce less FlaA protein at $37^{\circ} \mathrm{C}$ than at room temperature (Shen and Higgins 2006). Collectively, these results indicate that temperature-dependent, DegU-mediated transcriptional activation of 1 mo0688 allows for expression of flagellar motility genes specifically at low temperatures. Furthermore, additional post-transcriptional, temperature-dependent mechanisms regulate FlaA production and flagellar motility to restrict flagellar motility to low temperatures.

\section{Discussion}

Temperature-dependent expression of flagellar motility genes in L. monocytogenes is mediated by the opposing activities of MogR, a transcriptional repressor, and DegU, an indirect antagonist of $M o g R$. In this study, we identified a previously uncharacterized regulatory component that permits expression of flagellar motility genes at low temperatures. We demonstrate that MogR repression activity is inhibited at low temperatures by a MogR antirepressor, Lmo0688. Transcription of $1 m o 0688$ is controlled in a temperature-dependent manner by the DegU response regulator. Surprisingly, in addition to functioning as an anti-repressor for MogR, Lmo0688 is also a flagellin glycosyltransferase. Therefore, Lmo0688 transcriptionally regulates expression of FlaA, the substrate for its glycosyltransferase activity. Based on the bifunctionality of this protein, we have designated Lmo0688, $\mathrm{GmaR}$, to indicate its dual role as a glycosyltransferase and motility anti-repressor.

GmaR is the L. monocytogenes flagellin glycosyltransferase

Glycosylation is a common post-translational modification of bacterial flagella, yet the glycosyl moieties used by bacterial species vary extensively (Logan 2006).
Unique among prokaryotes, L. monocytogenes flagellin is modified by monomeric $\beta$-O-linked GlcNAc at three to six Ser/Thr residues (Schirm et al. 2004). While flagellar glycosylation in most bacterial species requires multiple genes dedicated specifically for synthesis of the unique glycan moiety, L. monocytogenes requires only UDP-GlcNAc, a common biosynthetic precursor (Szymanski and Wren 2005; Logan 2006). Thus, only a single enzyme with OGT activity would be required for FlaA glycosylation in L. monocytogenes (Schirm et al. 2004; Logan 2006).

In this study, we identified the L. monocytogenes OGT, GmaR, and characterized its function. By comparing a catalytically inactive glycosyltransferase mutant (D83N D85N) to wild-type GmaR using in vitro and in vivo studies, we demonstrate that GmaR has O-linked GlcNAc transferase activity (Fig. 4C,E). This represents the first flagellin glycosyltransferase for which glycosylation activity has been directly demonstrated. The discovery of GmaR is unique and significant, not only for its relevance to eukaryotic systems by being the first bacterial OGT identified and characterized to $\beta$-OGlcNAcylate a prokaryotic protein, but also for being the first flagellin glycosyltransferase to be identified and characterized in a Gram-positive organism.

\section{GmaR functions as an anti-repressor for $\operatorname{Mog} R$}

Our initial attempts to demonstrate that GmaR was the flagellin glycosyltransferase in $L$. monocytogenes were hindered by the fact that GmaR-negative bacteria fail to express FlaA. Surprisingly, this was due to a defect in flaA transcription rather than a defect in FlaA stability (Fig. 1C). Since transcriptional profiling of $\Delta g m a R$ showed complete repression of all flagellar motility genes at room temperature (Supplementary Table S1), and flagellar motility gene transcription was restored upon deletion of $\operatorname{mog} R$ in a GmaR-negative strain (Fig. $1 \mathrm{C})$, we determined that $\mathrm{GmaR}$ was required to relieve MogR-mediated repression. Interestingly, GmaR is the first prokaryotic glycosyltransferase to play a role in transcriptional regulation.

Although this is a novel role for a bacterial glycosyltransferase, OGTs in eukaryotic systems use the same $\beta$-O-linked GlcNAc moiety to modify and functionally alter proteins involved in transcriptional regulation (Jackson and Tjian 1988; Yang et al. 2002; Love and Hanover 2005). Therefore, we explored the possibility that glycosylation of either MogR or another unidentified substrate, resulted in antagonization of MogR repression. However, mutation of the glycosyltransferase domain of $\mathrm{GmaR}$ revealed that glycosylation was not required for the anti-repressor function of $\mathrm{GmaR}$ (Fig. 4D).

Since GmaR functions as an anti-repressor in the absence of its glycosyltransferase activity, it is evident that GmaR is a bifunctional protein with two distinct activities. The C-terminal domain of GmaR has little homology with any known proteins and may function sepa- 
rately from the $\mathrm{N}$-terminal glycosyltransferase domain. As determined by gel shift analysis and affinity purification studies (Fig. 5A,B), GmaR functions as an anti-repressor by forming a stable protein-protein complex with MogR that inhibits MogR DNA-binding activity. Anti-repression can occur through many mechanisms, but the GmaR:MogR system in L. monocytogenes may most closely resemble the CarS:CarA and SinI:SinR systems in Myxococcus xanthus and Bacillus subtilis, respectively (Bai et al. 1993; Scott et al. 1999; Whitworth and Hodgson 2001; Perez-Marin et al. 2004). Similar to the CarS:CarA system, GmaR is able to both prevent repressor binding as well as disrupt preformed repressorDNA complexes (Fig. 5A), indicating that MogR has a stronger affinity for GmaR than for its target DNA (Whitworth and Hodgson 2001). In M. xanthus, the CarS anti-repressor functions by interacting with the DNAbinding domain of CarA to hinder repressor activity (Perez-Marin et al. 2004). In contrast, the B. subtilis SinI anti-repressor prevents SinR from binding its target sites by disrupting SinR tetramer formation and forming an irreversible heteromultimer complex that is incapable of binding target DNA (Scott et al. 1999). Preliminary data suggest that MogR binds target DNA as a multimer (data not shown). Considering these two mechanisms, it is therefore conceivable that GmaR could either complex with the DNA-binding site of MogR as seen with CarS:CarA, or GmaR could prevent MogR multimer formation as seen with SinI:SinR.

The TPR repeat region of GmaR could mediate GmaR: MogR complex formation (Fig. 4A), since TPR repeats mediate protein-protein interactions and substrate specificity (Blatch and Lassle 1999; Iyer and Hart 2003). Interestingly, the TPR domain of human OGT is responsible for targeting OGT to a transcriptional repressor complex (Yang et al. 2002; Iyer and Hart 2003). Alternatively, the TPR region of GmaR could mediate interactions with FlaA, the glycosyltransferase substrate. Functional domain mapping of both GmaR and MogR is currently underway to determine the role of the TPR region in mediating either glycosyltransferase or anti-repressor activity and to further understand the nature of the GmaR:MogR interaction.

The DegU response regulator mediates temperaturedependent control of flagellar motility gene expression

If the GmaR:MogR complex inactivates MogR-mediated repression of flagellar motility gene expression, what determines the temperature specificity of this interaction? Since MogR protein levels are temperature independent (Shen and Higgins 2006), we determined that the GmaR: MogR complex must occur only at low temperatures. In this study, we reveal that transcription of gmaR is temperature dependent, making GmaR available only at low temperatures to antagonize MogR (Fig. 6A,B). This environmental control of anti-repressor expression is also seen in the SinI:SinR system, where the SinR repressor is constitutively expressed and the SinI anti-repressor is expressed only under sporulation conditions (Bai et al. 1993).

Since previous studies revealed that the DegU response regulator was also required to antagonize MogR repression (Shen and Higgins 2006), we examined whether DegU mediates temperature-dependent control of GmaR expression. Epistasis analysis revealed that gmaR is both DegU-activated and MogR-repressed (Fig. 6A). Constitutive expression of GmaR in a DegU-negative strain revealed that GmaR functions downstream from DegU as a DegU-regulated factor required for flagellar gene expression (Fig. 3A). Since GmaR can be biologically active as an anti-repressor at $37^{\circ} \mathrm{C}$ (Fig. $6 \mathrm{C}$ ), it is the DegUmediated control of gmaR transcription that confers temperature specificity to flagellar gene expression. Microarray analyses comparing the transcriptional profile of $\Delta \operatorname{mog} R \Delta \operatorname{deg} U$ to $\Delta \operatorname{mog} R$ indicated that $\operatorname{DegU}$ is also required for transcriptional activation of flagellar genes located upstream of gmaR (data not shown). This observation suggests that critical components of the flagellar apparatus are absent in a $\Delta \operatorname{deg} U$ strain, resulting in the lack of motility observed in $\Delta$ degU/cgmaR (Fig. 3C). Specifically, although heterologous expression of GmaR in $\Delta d \operatorname{deg} U$ antagonizes $\operatorname{Mog} \mathrm{R}$ repression activity to restore FlaA expression, GmaR expression is not sufficient to activate transcription of additional DegU-regulated flagellar genes required for proper assembly of flagella. Studies are currently underway to identify the DegUregulated promoter(s) and the mechanism controlling temperature-dependent transcriptional activation by DegU. Furthermore, aside from the proposed requirement for DegU to activate transcription of a subset of flagellar genes, an additional post-transcriptional mechanism appears to regulate flagellar motility during growth at $37^{\circ} \mathrm{C}$. Even when flagellar motility gene transcription is artificially induced at $37^{\circ} \mathrm{C}$ by the constitutive expression of GmaR in $\Delta g m a R / \operatorname{cgmaR}$ (Fig. 6C) and $\Delta \operatorname{mog} R$ (Shen and Higgins 2006), FlaA protein levels are dramatically reduced.

Our results demonstrate that the DegU response regulator, the motility gene repressor MogR, and the bifunctional glycosyltransferase/anti-repressor GmaR comprise the molecular circuitry that mediates temperaturedependent regulation of flagellar motility gene expression in L. monocytogenes. In Figure 7, we depict a working model for $L$. monocytogenes flagellar gene expression using the fla $A$ promoter region as an example. At physiological temperatures $\left(37^{\circ} \mathrm{C}\right.$ and higher), MogR completely represses flagellar gene expression by binding promoter region DNA, resulting in nonflagellated, nonmotile bacteria. As the temperature decreases, DegU either directly or indirectly activates gmaR transcription. Once gmaR is transcribed and translated, GmaR sequesters MogR in a GmaR:MogR complex, relieving repression of its own promoter and other flagellar gene promoters. Subsequent to production of FlaA, GmaR functions as a $\beta$-O-linked GlcNAc transferase mediating glycosylation of FlaA. Thus, GmaR functions as an anti-repressor that permits expression of FlaA, the substrate for its glycosyltransferase activity. 


\begin{abstract}
Figure 7. Working model for temperature-dependent regulation of flagellar motility gene expression in L. monocytogenes. At $37^{\circ} \mathrm{C}$ and above, MogR represses transcription by binding to target sequences in promoter region DNA (flaA and gmaR shown). As temperature decreases, DegU activates transcription of gmaR through an unknown mechanism (lightning bolt). GmaR removes MogR bound to target sequences by protein-protein interaction, alleviating repression of flagellar motility gene promoters. Antirepression results in the production of the flagellin monomer (FlaA). GmaR glycosylates FlaA with $\beta$-Olinked GlcNAc (stars). The gmaR coding region is represented by an open arrow, and transcription initiating from an uncharacterized $g m a R$ promoter is indicated by a dashed bent arrow. The fla $\mathrm{A}$ coding region is represented by a gray arrow, and transcription initiating from the flaA promoter is indicated by a solid bent arrow. A solid line indicates repression occurring by MogR binding to target sequences (open boxes). This model is representative of events occurring at all flagellar motility gene promoter regions.
\end{abstract}

$37^{\circ} \mathrm{C}$ and above

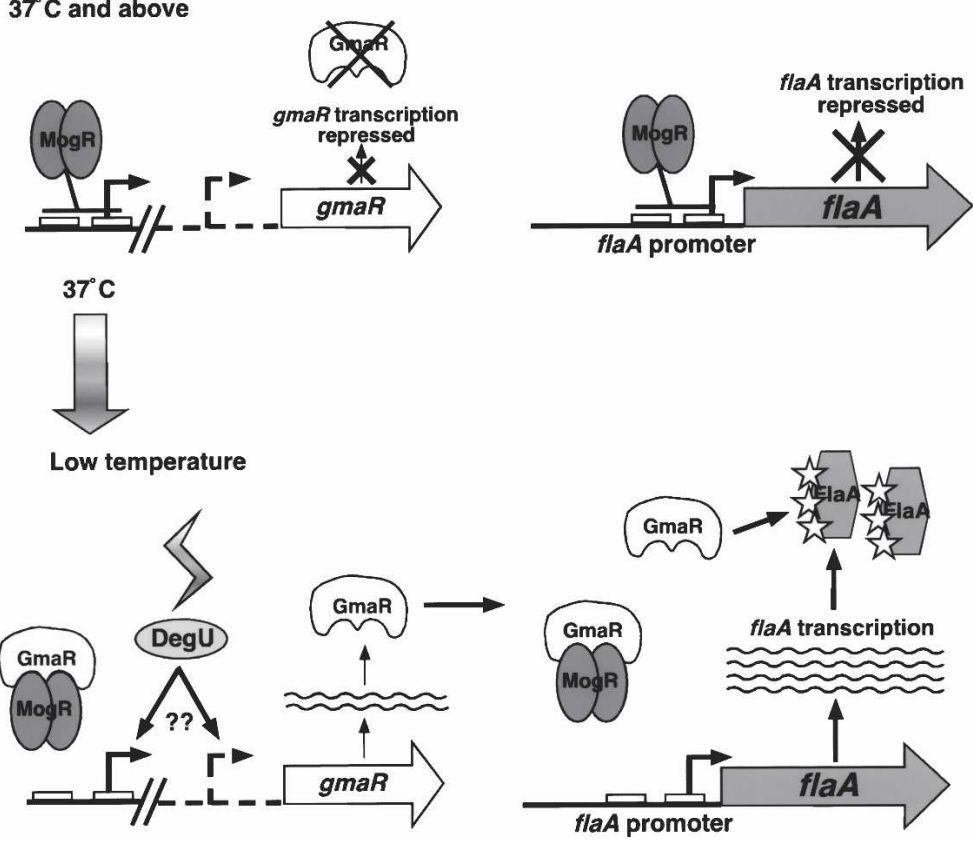

Role of flagellin glycosylation in L. monocytogenes

In some bacterial species, glycosylation has a significant role in the regulation of flagellar motility (for review, see Logan 2006). L. monocytogenes, however, is unlike any prokaryotic organism studied to date, since deletion of the flagellin glycosyltransferase results in a transcriptional defect rather than a post-transcriptional defect (Fig. 1C). Our results demonstrate that GmaR glycosyltransferase function is independent from its anti-repressor activity, since inactivation of glycosyltransferase activity by mutation has no effect on flagellar motility under the conditions examined (Fig. 4D). Therefore, unlike other organisms, the secretion and stability of $L$. monocytogenes flagellin is not inherently dependent upon glycosylation.

Although a significant number of secreted prokaryotic appendages, such as flagella and pili, are modified by glycosylation, the specific role for glycosylation in prokaryotic systems remains elusive. Similar to L. monocytogenes, glycosylation mutants in Pseudomonas aeruginosa secrete unglycosylated flagellin that is assembled into fully functional flagella (Arora et al. 2005). These glycosylation mutants are defective in their induction of innate immune responses and attenuated in a burnedmouse model of infection, implicating a role for glycosylation in P. aeruginosa pathogenesis (Arora et al. 2005; Verma et al. 2005). However, since flagella expression is repressed at physiological temperatures in L. monocytogenes and has been shown to be dispensable for virulence (Way et al. 2004), it is unlikely that glycosylation plays a role in L. monocytogenes infection or immune evasion. In support of this hypothesis, preliminary studies indicate that purified flagella from wild type (glycosylated) and $\Delta \operatorname{mog} R \Delta g m a R$ (unglycosylated) do not differentially activate NF-кB (data not shown). However, L. monocy- togenes has the ability to thrive in a diverse range of biological niches, and glycosylation of flagella may be an important factor for environmental adaptation outside the host.

In this study, we provide the first example of a bifunctional prokaryotic flagellar OGT that controls expression of its glycosyltransferase substrate by anti-repression. By linking OGT activity and anti-repression function together in one protein, this system is primed to exclusively produce glycosylated flagella. While there exists several examples of bifunctional enzyme/transcription factors (Min et al. 1993; Ostrovsky de Spicer and Maloy 1993), GmaR is a unique example of an antirepressor that controls the transcription of its own enzymatic substrate. It is interesting to speculate that by virtue of its ability to bind $\operatorname{Mog} \mathrm{R}$, which itself binds the flaA promoter, GmaR may become spatially localized near the site of production of its enzymatic substrate, FlaA. Thus, the bifunctionality of GmaR may confer the spatial coordination required to ensure maximal glycosylation of the flagellin subunit in L. monocytogenes.

\section{Materials and methods}

Strain construction and culture conditions

Antibiotics were used at the following concentrations: chloramphenicol $20 \mu \mathrm{g} / \mathrm{mL}$ for selection of pPL2 derivatives in E. coli and $7.5 \mu \mathrm{g} / \mathrm{mL}$ for selection of integrated pPL2 derivatives and transformed pCON1 strains in L. monocytogenes; $100 \mu \mathrm{g} / \mathrm{mL}$ carbenicillin for pCON1 derivatives in E. coli; $30 \mu \mathrm{g} / \mathrm{mL}$ kanamycin for $\mathrm{pET}$ vectors in $E$. coli; and $1 \mu \mathrm{g} / \mathrm{mL}$ erythromycin for selection of $L$. monocytogenes strains with Tn917-derived transposon insertions. Strains used in this study are listed in Supplementary Table S2, and primers are listed in Supplementary Table S3. Strain constructions are described in the Supple- 
mental Material. All plasmid constructs were confirmed by automated sequencing.

\section{Motility assay}

A single colony was inoculated with a straight needle into BHI agar $(0.375 \%)$. Motility was assessed after $48 \mathrm{~h}$ incubation at room temperature.

\section{Microarray analysis}

Microarray analyses were performed as previously described (Shen and Higgins 2006), except that the threshold for considering a gene as Lmo0688 regulated was set at a fold change $>2.5$ with $p<0.02$. For hierarchal clustering, the Rosetta Resolver Euclidean clustering algorithm was employed to compute the dendrogram (Fig. 2).

\section{In vitro O-GlcNAc transferase assay}

Fourteen-hour to 16 -h cultures of $\Delta \operatorname{mog} R \Delta 688 \Delta f l a A$ and $\Delta \operatorname{mog} R \Delta 688$ were diluted 1:30 into $30 \mathrm{~mL}$ of $\mathrm{BHI}$ and grown for $4 \mathrm{~h}$ at $28^{\circ} \mathrm{C}$ with shaking. Bacteria were pelleted, resuspended in $300 \mu \mathrm{L}$ Listeria lysis buffer $(50 \mathrm{mM}$ Tris at $\mathrm{pH} 7.5,100 \mathrm{mM}$ $\mathrm{NaCl}, 1 \mathrm{mM}$ DTT, $10 \%$ glycerol, $1 \mathrm{mM} \mathrm{MgCl}_{2}$, CompleteEDTA protease inhibitor mixture [Roche], $1 \mathrm{mg} / \mathrm{mL}$ lysozyme), and disrupted by mechanical lysis in Fast ProBlue tubes using the FastPrep apparatus (Qbiogene) according to the manufacturer's specifications. The lysate was cleared by centrifugation. One microgram of purified $\mathrm{His}_{6}$-tagged Lmo0688 or $\mathrm{His}_{6}$-tagged D83N D85N, $0.5 \mu \mathrm{Ci}$ of $\left[{ }^{14} \mathrm{C}\right]$-uridine diphosphate $N$-acetyl-Dglucosamine ([ $\left.{ }^{14} \mathrm{C}\right]-U D P-G l c N A c ;$ Perkin-Elmer), and $50 \mu \mathrm{L}$ of L. monocytogenes cell extracts (equivalent to $\sim 4 \mathrm{~mL}$ of culture) were mixed together with lysis buffer for a total volume of 100 $\mu \mathrm{L}$. The reaction was allowed to proceed at $37^{\circ} \mathrm{C}$, during which time $25-\mu \mathrm{L}$ aliquots were removed and added to $25 \mu \mathrm{L}$ of $2 \times \mathrm{FSB}$ at the time points indicated. The samples were boiled and resolved on a $12 \%$ SDS-PAGE gel. The resulting gel was processed for enhanced autoradiography using EN3HANCE (PerkinElmer) according to the manufacturer's specifications.

\section{Gel mobility shift analysis}

Gel shift analysis was performed as previously described (Shen and Higgins 2006). For the binding reactions containing MogR and either Lmo0688 or DegU, 0.1 pmol of DNA probe was incubated with 0.8 pmol of $\mathrm{His}_{6}$-tagged MogR protein in $1 \times \mathrm{BB}$ for 30 min, then $\mathrm{His}_{6}$-tagged Lmo0688 or $\mathrm{His}_{6}$-tagged DegU was added at the indicated amount, and the entire reaction was incubated for an additional $30 \mathrm{~min}$ at $30^{\circ} \mathrm{C}$. Reactions containing either $\mathrm{His}_{6}$-tagged Lmo0688 or $\mathrm{His}_{6}$-tagged $\mathrm{MogR}$ alone were incubated with $0.1 \mathrm{pmol}$ of DNA probe for $30 \mathrm{~min}$ at $30^{\circ} \mathrm{C}$. Binding reactions were analyzed as previously described (Shen and Higgins 2006).

\section{Affinity pull-down assays}

Affinity pull-down assays were performed using purified $\mathrm{His}_{6}{ }^{-}$ tagged Lmo0688 and L. monocytogenes cell lysates. To prepare cell lysates, $1 \mathrm{~mL}$ of a 14-16-h culture grown at room temperature was used to inoculate $100 \mathrm{~mL}$ BHI. The $100-\mathrm{mL}$ BHI cultures were grown without shaking for $18-20 \mathrm{~h}$ at room temperature in 1-L flasks. Cultures were pelleted at $6500 \times \mathrm{g}$ for $10 \mathrm{~min}$, and the pellet was resuspended in $4 \mathrm{~mL}$ of $\mathrm{PB}$ buffer $(10 \mathrm{mM}$ Tris at $\mathrm{pH} 7.5,6 \mathrm{mM}$ imidazole, $100 \mathrm{mM} \mathrm{NaCl}, 10 \%$ glycerol, $0.5 \mathrm{mM}$ DTT). Samples were processed for lysis in $1-\mathrm{mL}$ volumes using FastProtein Blue tubes and a FastPrep apparatus
(Qbiogene). Each 1-mL aliquot was processed for $20 \mathrm{sec}$ at setting 6.0 and then placed on ice for $2 \mathrm{~min}$, and the procedure was repeated three times. Bacterial cell extracts were recovered by pelleting the lysis matrix by centrifugation at $16,000 \times g$ for 20 min at $4^{\circ} \mathrm{C}$. The recovered supernatant was then centrifuged an additional $10 \mathrm{~min}$ at $16,000 \times g$ at $4^{\circ} \mathrm{C}$. Supernatant samples were pooled. To prepare the Ni-NTA agarose, $1 \mathrm{~mL}$ of Ni-NTA agarose (Qiagen) was pelleted at $1000 \times g$ for $1 \mathrm{~min}$, washed three times with $1 \mathrm{~mL}$ of $\mathrm{PB}$, and resuspended in a final volume of $1 \mathrm{~mL}$ of PB. To each $1-\mathrm{mL}$ volume of lysate, $100 \mu \mathrm{L}$ of washed Ni-NTA agarose and $3 \mu$ of His $_{6}$-tagged Lmo0688 were added. Pull-down reactions were incubated on a rotator platform for $6 \mathrm{~h}$ at $4^{\circ} \mathrm{C}$. Samples were pelleted at $1000 \times g$ and washed twice in $1 \mathrm{~mL}$ of $\mathrm{PB}$, incubated rotating for $10 \mathrm{~min}$ at $4^{\circ} \mathrm{C}$, and then washed three times with $1 \mathrm{~mL}$ of $\mathrm{PB}$. After a final centrifugation, the Ni-NTA beads and bound sample were resuspended in $80 \mu \mathrm{L}$ of $2 \times$ loading buffer, boiled for $3 \mathrm{~min}$, and centrifuged at $2000 \times g$ for $1 \mathrm{~min}$, and $65 \mu \mathrm{L}$ was loaded onto a $10 \%$ SDS-PAGE gel. Western blot analysis was performed using either a MogRspecific antibody or an Lmo0688-specific antibody.

\section{$\beta$-galactosidase measurement of flaA promoter activity}

$\beta$-galactosidase assays were performed as previously described (Shen and Higgins 2006). The flaA promoter-lacZ reporter fusion (flaA::Tn) consists of a Tn917-lacZ transposon inserted within the fla $A$ gene at nucleotide 117 . However, the lacZ gene contains an associated ribosome-binding site.

\section{Data deposition}

The microarray data sets and Rosetta Resolver analyses reported in this paper have been deposited in the Gene Expression Omnibus database under accession number GSE6032 (http://www. ncbi.nlm.nih.gov/geo/query/acc.cgi?acc=GSE6032).

\section{Supplemental Material}

Supplemental Material includes additional experimental procedures, two figures, and three tables.

\section{Acknowledgments}

We thank Christine Alberti-Segui for construction of the pHLIV2 vector and Benjamin Gross for helpful discussions regarding OGT and providing advice and reagents for the in vitro glycosylation assays. We are indebted to Joseph Mougous for help with protein purifications, providing invaluable insights into the development of this project, and for critical reading of the manuscript. We also thank Ann Hochschild for critical review of the manuscript. Appreciation is given to the Pathogen Functional Genomic Resource Center at TIGR for providing the microarrays used in this study. This work was supported by U.S. Public Health Service grant AI53669 from the National Institutes of Health (to D.E.H.). A.S. is a recipient of a Howard Hughes predoctoral fellowship award.

\section{References}

Akerley, B.J. and Miller, J.F. 1993. Flagellin gene transcription in Bordetella bronchiseptica is regulated by the BvgAS virulence control system. J. Bacteriol. 175: 3468-3479.

Akerley, B.J., Cotter, P.A., and Miller, J.F. 1995. Ectopic expression of the flagellar regulon alters development of the Bordetella-host interaction. Cell 80: 611-620. 
Aldridge, P. and Hughes, K.T. 2002. Regulation of flagellar assembly. Curr. Opin. Microbiol. 5: 160-165.

Arora, S.K., Neely, A.N., Blair, B., Lory, S., and Ramphal, R. 2005. Role of motility and flagellin glycosylation in the pathogenesis of Pseudomonas aeruginosa burn wound infections. Infect. Immun. 73: 4395-4398.

Bai, U., Mandic-Mulec, I., and Smith, I. 1993. SinI modulates the activity of SinR, a developmental switch protein of $\mathrm{Ba}$ cillus subtilis, by protein-protein interaction. Genes \& Dev. 7: 139-148.

Blatch, G.L. and Lassle, M. 1999. The tetratricopeptide repeat: A structural motif mediating protein-protein interactions. Bioessays 21: 932-939.

Campbell, J.A., Davies, G.J., Bulone, V., and Henrissat, B. 1997. A classification of nucleotide-diphospho-sugar glycosyltransferases based on amino acid sequence similarities. Biochem. J. 326: 929-939.

Comer, F.I., Vosseller, K., Wells, L., Accavitti, M.A., and Hart, G.W. 2001. Characterization of a mouse monoclonal antibody specific for O-linked N-acetylglucosamine. Anal. Biochem. 293: 169-177.

Eisen, M.B., Spellman, P.T., Brown, P.O., and Botstein, D. 1998. Cluster analysis and display of genome-wide expression patterns. Proc. Nat1. Acad. Sci. 95: 14863-14868.

Goon, S., Kelly, J.F., Logan, S.M., Ewing, C.P., and Guerry, P. 2003. Pseudaminic acid, the major modification on Campylobacter flagellin, is synthesized via the Cj1293 gene. Mol. Microbiol. 50: 659-671.

Gründling, A., Burrack, L.S., Bouwer, H.G., and Higgins, D.E. 2004. Listeria monocytogenes regulates flagellar motility gene expression through $\operatorname{Mog} \mathrm{R}$, a transcriptional repressor required for virulence. Proc. Natl. Acad. Sci. 101: 1231812323.

Hayashi, F., Smith, K.D., Ozinsky, A., Hawn, T.R., Yi, E.C., Goodlett, D.R., Eng, J.K., Akira, S., Underhill, D.M., and Aderem, A. 2001. The innate immune response to bacterial flagellin is mediated by Toll-like receptor 5. Nature 410: 1099-1103.

Hughes, E.A. and Galan, J.E. 2002. Immune response to Salmonella: Location, location, location? Immunity 16: 325-328.

Iyer, S.P. and Hart, G.W. 2003. Roles of the tetratricopeptide repeat domain in O-GlcNAc transferase targeting and protein substrate specificity. I. Biol. Chem. 278: 2460824616.

Jackson, S.P. and Tjian, R. 1988. O-glycosylation of eukaryotic transcription factors: Implications for mechanisms of transcriptional regulation. Cell 55: 125-133.

Josenhans, C., Vossebein, L., Friedrich, S., and Suerbaum, S. 2002. The neuA/flmD gene cluster of Helicobacter pylori is involved in flagellar biosynthesis and flagellin glycosylation. FEMS Microbiol. Lett. 210: 165-172.

Kapatral, V., Olson, J.W., Pepe, J.C., Miller, V.L., and Minnich, S.A. 1996. Temperature-dependent regulation of Yersinia enterocolitica class III flagellar genes. Mol. Microbiol. 19: 1061-1071.

Knudsen, G.M., Olsen, J.E., and Dons, L. 2004. Characterization of DegU, a response regulator in Listeria monocytogenes, involved in regulation of motility and contributes to virulence. FEMS Microbiol. Lett. 240: 171-179.

Logan, S.M. 2006. Flagellar glycosylation-A new component of the motility repertoire? Microbiol. 152: 1249-1262.

Logan, S.M., Kelly, J.F., Thibault, P., Ewing, C.P., and Guerry, P. 2002. Structural heterogeneity of carbohydrate modifications affects serospecificity of Campylobacter flagellins. Mol. Microbiol. 46: 587-597.

Love, D.C. and Hanover, J.A. 2005. The hexosamine signaling pathway: Deciphering the 'O-GlcNAc code.' Sci. STKE 2005: re13.

Lubas, W.A. and Hanover, J.A. 2000. Functional expression of O-linked GlcNAc transferase. Domain structure and substrate specificity. J. Biol. Chem. 275: 10983-10988.

Macnab, R.M. 2003. How bacteria assemble flagella. Annu. Rev. Microbiol. 57: 77-100.

Min, K.T., Hilditch, C.M., Diederich, B., Errington, J., and Yudkin, M.D. 1993. $\sigma^{\mathrm{F}}$, the first compartment-specific transcription factor of $B$. subtilis, is regulated by an anti- $\sigma$ factor that is also a protein kinase. Cell 74: 735-742.

Molofsky, A.B., Byrne, B.G., Whitfield, N.N., Madigan, C.A., Fuse, E.T., Tateda, K., and Swanson, M.S. 2006. Cytosolic recognition of flagellin by mouse macrophages restricts $L e$ gionella pneumophila infection. J. Exp. Med. 203: 10931104.

Ostrovsky de Spicer, P. and Maloy, S. 1993. PutA protein, a membrane-associated flavin dehydrogenase, acts as a redoxdependent transcriptional regulator. Proc. Natl. Acad. Sci. 90: 4295-4298.

Ott, M., Messner, P., Heesemann, J., Marre, R., and Hacker, J. 1991. Temperature-dependent expression of flagella in Legionella. J. Gen. Microbiol. 137: 1955-1961.

Peel, M., Donachie, W., and Shaw, A. 1988. Temperature-dependent expression of flagella of Listeria monocytogenes studied by electron microscopy, SDS-PAGE and Western blotting. J. Gen. Microbiol. 134: 2171-2178.

Perez-Marin, M.C., Lopez-Rubio, J.J., Murillo, F.J., Elias-Arnanz, M., and Padmanabhan, S. 2004. The $\mathrm{N}$ terminus of Myxococcus xanthus CarA repressor is an autonomously folding domain that mediates physical and functional interactions with both operator DNA and antirepressor protein. J. Biol. Chem. 279: 33093-33103.

Ramos, H.C., Rumbo, M., and Sirard, J.C. 2004. Bacterial flagellins: Mediators of pathogenicity and host immune responses in mucosa. Trends Microbiol. 12: 509-517.

Ren, T., Zamboni, D.S., Roy, C.R., Dietrich, W.F., and Vance, R.E. 2006. Flagellin-deficient Legionella mutants evade caspase-1- and Naip5-mediated macrophage immunity. PLoS Pathog. 2: e18.

Schirm, M., Soo, E.C., Aubry, A.J., Austin, J., Thibault, P., and Logan, S.M. 2003. Structural, genetic and functional characterization of the flagellin glycosylation process in Helicobacter pylori. Mol. Microbiol. 48: 1579-1592.

Schirm, M., Kalmokoff, M., Aubry, A., Thibault, P., Sandoz, M., and Logan, S.M. 2004. Flagellin from Listeria monocytogenes is glycosylated with $\beta$-O-linked $\mathrm{N}$-acetylglucosamine. J. Bacteriol. 186: 6721-6727.

Scott, D.J., Leejeerajumnean, S., Brannigan, J.A., Lewis, R.J., Wilkinson, A.J., and Hoggett, J.G. 1999. Quaternary re-arrangement analysed by spectral enhancement: The interaction of a sporulation repressor with its antagonist. J. Mol. Biol. 293: 997-1004.

Shen, A. and Higgins, D.E. 2006. The MogR transcriptional repressor regulates non-hierarchal expression of flagellar motility genes and virulence in Listeria monocytogenes. PLoS Pathog. 2: e30.

Szymanski, C.M. and Wren, B.W. 2005. Protein glycosylation in bacterial mucosal pathogens. Nat. Rev. Microbiol. 3: 225237.

Takeuchi, K., Taguchi, F., Inagaki, Y., Toyoda, K., Shiraishi, T., and Ichinose, Y. 2003. Flagellin glycosylation island in Pseudomonas syringae pv. glycinea and its role in host specificity. J. Bacteriol. 185: 6658-6665.

Unligil, U.M., Zhou, S., Yuwaraj, S., Sarkar, M., Schachter, H., and Rini, J.M. 2000. X-ray crystal structure of rabbit 
$\mathrm{N}$-acetylglucosaminyltransferase I: Catalytic mechanism and a new protein superfamily. EMBO J. 19: 5269-5280.

Vatanyoopaisarn, S., Nazli, A., Dodd, C.E., Rees, C.E., and Waites, W.M. 2000. Effect of flagella on initial attachment of Listeria monocytogenes to stainless steel. Appl. Environ. Microbiol. 66: 860-863.

Verma, A., Arora, S.K., Kuravi, S.K., and Ramphal, R. 2005. Roles of specific amino acids in the $\mathrm{N}$ terminus of Pseudomonas aeruginosa flagellin and of flagellin glycosylation in the innate immune response. Infect. Immun. 73: 8237-8246.

Way, S.S., Thompson, L.J., Lopes, J.E., Hajiar, A.M., Kollmann, T.R., Freitag, N.E., and Wilson, C.B. 2004. Characterization of flagellin expression and its role in Listeria monocytogenes infection and immunity. Cell. Microbiol. 6: 235-242.

Whitworth, D.E. and Hodgson, D.A. 2001. Light-induced carotenogenesis in Myxococcus xanthus: Evidence that CarS acts as an anti-repressor of CarA. Mol. Microbiol. 42: 809819.

Williams, T., Joseph, B., Beier, D., Goebel, W., and Kuhn, M. 2005. Response regulator DegU of Listeria monocytogenes regulates the expression of flagella-specific genes. FEMS Microbiol. Lett. 252: 287-298.

Yang, X., Zhang, F., and Kudlow, J.E. 2002. Recruitment of OGlcNAc transferase to promoters by corepressor mSin3A: Coupling protein O-GlcNAcylation to transcriptional repression. Cell 110: 69-80.

Zhang, F., Su, K., Yang, X., Bowe, D.B., Paterson, A.J., and Kudlow, J.E. 2003. O-GlcNAc modification is an endogenous inhibitor of the proteasome. Cell 115: 715-725. 


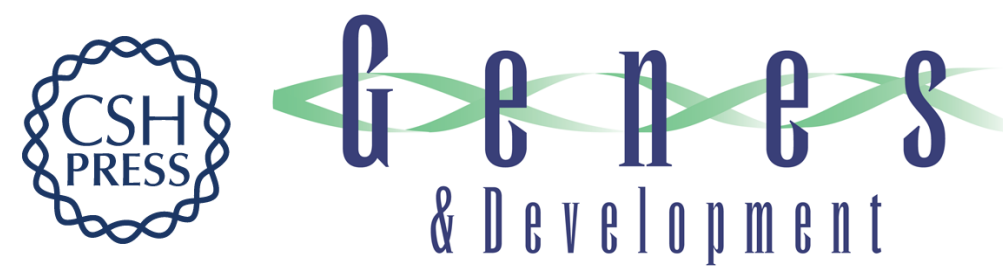

\section{A bifunctional O-GIcNAc transferase governs flagellar motility through anti-repression}

Aimee Shen, Heather D. Kamp, Angelika Gründling, et al.

Genes Dev. 2006, 20:

Access the most recent version at doi:10.1101/gad.1492606

\section{Supplemental http://genesdev.cshlp.org/content/suppl/2006/11/16/20.23.3283.DC1 Material}

References This article cites 43 articles, 15 of which can be accessed free at: http://genesdev.cshlp.org/content/20/23/3283.full.html\#ref-list-1

\section{License}

Email Alerting

Receive free email alerts when new articles cite this article - sign up in the box at the top Service 